\title{
Acute myeloid leukemia presenting with panhypopituitarism and diabetes insipidus
}

\author{
Fulya Akin ${ }^{1}$,Şenay Topsakal ${ }^{1}$,Veysel Erol ${ }^{2}$,Guzin Fidan Yaylali ${ }^{1}$, Yilmaz Kiroglu ${ }^{3}$, Mehmet Sercan Erturk $^{1}$
}

\author{
${ }^{1}$ Pamukkale University, Faculty of Medicine, Department of Endocrinology and Metabolism, Denizli, \\ 20070, Turkey \\ ${ }^{2}$ Pamukkale University, Faculty of Medicine, Department of Internal Medicine,Denizli,20070, Turkey \\ ${ }^{3}$ Pamukkale University, Faculty of Medicine, Department of Radiology, Denizli, 20070, Turkey
}

\section{Introduction}

Central nervous system involvement is rare in acute myeloid leukemia (AML) cases. Pituitary involvement is much more rarely seen with unknown frequency and if left untreated, may result in death. Although rarely seen, leukemic infiltration of the pituitary gland should be evaluated in leukemic patients with visual disturbance, hypopituitarism or central diabetes insipidus(CDI). To date, AML presenting with CDI has been rarely reported. The presence of both anterior and posterior pituitary deficiency is even rarer with unknown prevalence. Here, we present a patient with AML (subtype clasification is proceeding ) presenting with panhypopituitarism and CDI.

\section{Case report}

A 37 years old man, with no significant past medical history, presented to emergency department with complaint of high fever, polyuria and polydipsia Laboratory tests revealed increased CRP, ESR, serum sodium was 157 $\mathrm{mEq} / \mathrm{L}$.Patient diagnosed as panhypopituitarism according to the pituitary hormones values ( TSH:0.04 ulU/mL (0,2-4,2), ft4:0.801 ng/dL(0,99-1,65), kortizol:3,21 ug/dL, ACTH:12,3 pg/mL ). On the cranial mri, FLAIR image shows hyperintense lesions in bilateral hypothalamic areas probable due to leukemic infiltration(image 1).contrastenhanced T1W-coronal image demonstrates rim enhancement pattern(image 2), $\mathrm{mr}$ spectroscopy reveals high cho/cr and cho/naa ratios(image 3-4). Patient admitted the endocrinology clinic and hyponatremic IV hydration $(\% 0,45$ saline and $\% 5$ dextroz) was given in order. After the initiation of this treatment, urine output was noticed to be 6000-7000 cc per day. Water deprivation test showed worsening of hypernatremia (sodium: $151 \mathrm{mEq} / \mathrm{L}$ ), and serum osmolality test showed hyperosmolality $(312 \mathrm{mosm} / \mathrm{kg}$ ) with inappropriate low urine osmolality (130 $\mathrm{mOsm} / \mathrm{kg}$ ). Urinary osmolality was increased by two times following vasopressin administration. Nasal 1-deamino-8-D-arginine vasopressin (DDAVP) was started with the diagnosis of central diabetes insipidus. Polyuria and polydipsia were improved under DDAVP treatment and correction of hypernatremia and dehydration,Patient was treated with prednizolon, desmopression and levothyroxine sodium for panhypopituitarism. On the third day of admission, the laboratory studies showed neutropenia (white blood cell $2.6 \mathrm{~K} / \mu \mathrm{L}$ ), macrocytic anaemia (Hemoglobin $10.2 \mathrm{~g} / \mathrm{dL}, \mathrm{MCV} 131.7 \mathrm{fL}$ ), and mild thrombocytosis. Patient family history was noncontributory. The detailed history showed that the patient had unintentional weight loss of 20 pounds in the past 1 month. The physical examination showed pale conjunctivae. No other significant abnormality was noticed on examination. Examination of a peripheral blood smear revealed moderate normochromic normocytic anemia with no rouleaux formation. Mild anisocytosis and occasional oval macrocytes were noted. The majority of nucleated blood cells were large with myeloid features with a few atypical blastic cells. The serum total protein was decreased at $52 \mathrm{~g} / \mathrm{L}$ (reference range 65-85) and beta2-microglobulin elevated at $6.3 \mathrm{mg} / \mathrm{L}$ (reference range $<2$ ). Serum immunoglobulin levels were in normal range, with lgG:1306 mg/dl, IgA:170 mg/dl, IgM:214 mg/dl, kappa light chain:347,lambda light chain:205 mg/dl. A smear of bone marrow aspirate showed sheets of myeloid cells that accounted for $82 \%$ of the overall nucleated cell population. Large, binucleate and trinucleate forms were noted. Many of these atypical myeloid cells contained small, inconspicuous nucleoli. A diagnosis of myeloid leukemia was made by hematology. Patient will follow up hematology clinic for further investigation and treatments.

\section{Discussion}

CDI in patients with $A M L$ is uncommon with a prevalence of less than $1 \%$. It may occur at the same time or after the diagnosis of hematological disease. CDI associated with AML may develop due to leukemic infiltration and different mechanisms such as chromosomal abnormality, dysmegakaryopoiesis and thrombocytosis. Monosomy 7 is the most common cytogenetic chromosomal abnormality determined in these cases. It has been shown that the presence of $\mathrm{DI}$ and monosomy 7 was associated with higher mortality rates than DI without this abnormality. The other chromosomal abnormality is structural abnormalities on the long arm of chromosome 3 . In genetic analyses, there was no chromosomal abnormality in our patient. Lavabre-Bertrand et al. described trombocytosis in three patients with $\mathrm{AML}$ and $\mathrm{DI}$, all showing monosomy 7 and chromosome 3 abnormalities. Interestingly, these three cases had a normal computed tomography scan of the brain. The authors stated that this might be a new disease entity. Although our patient had slightly elevated platelet count, there wasn't pituitary involvement by leukemic cells on MRI like previous cases. When pituitary involvement detected, hormone function tests are required for both anterior and posterior lobes of the pituitary gland. Adrenal insufficiency and hypothyroidism may mask diabetes insipidus, thus, steroid and thyroxin treatment can cause diabetes insipidus. We found only three cases of AML presenting with panhypopituitarism and DI in the literature. However, subtypes of AML were not stated in those reports except one case due to acute myelomonocytic leukemia.
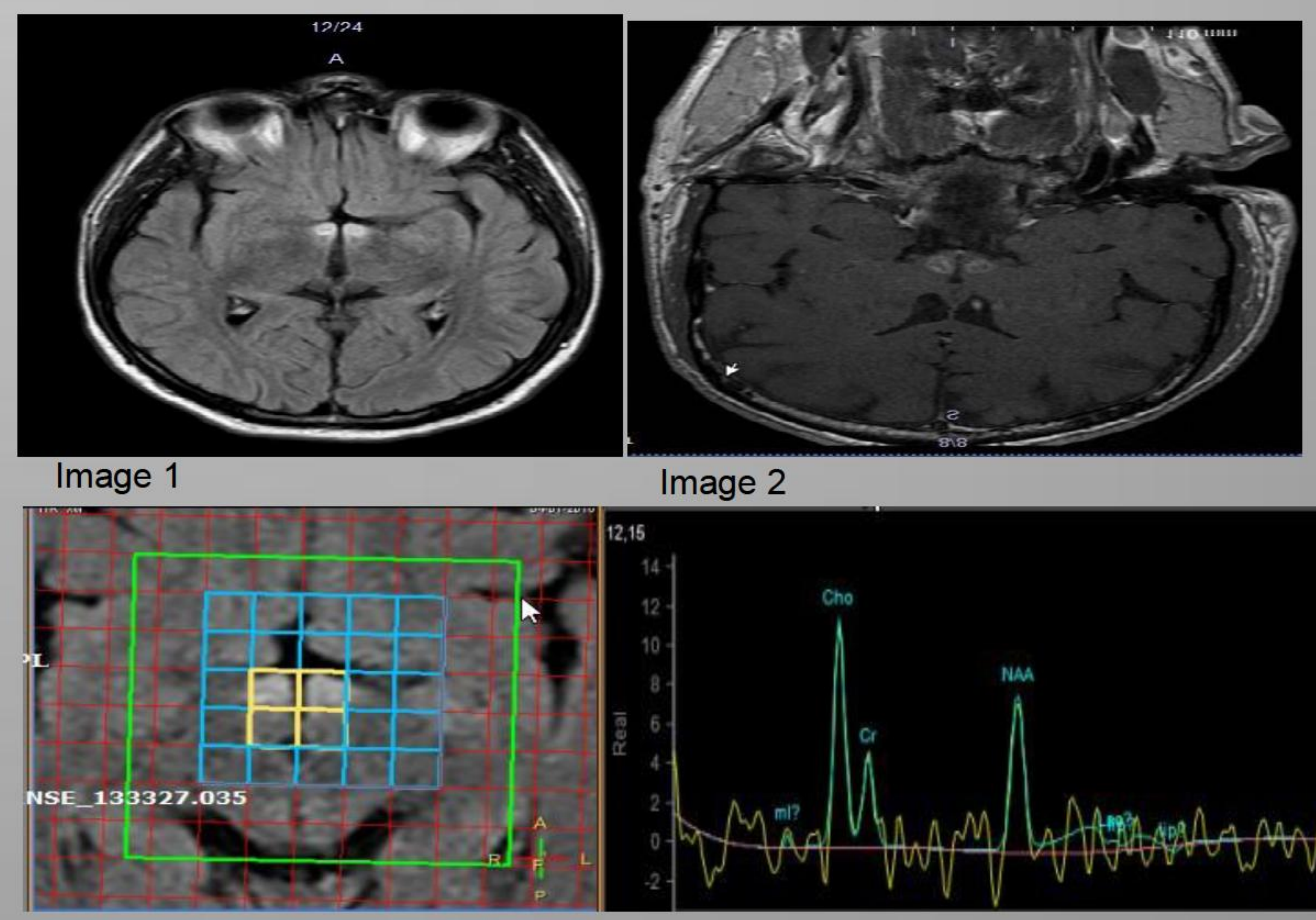

Image 2

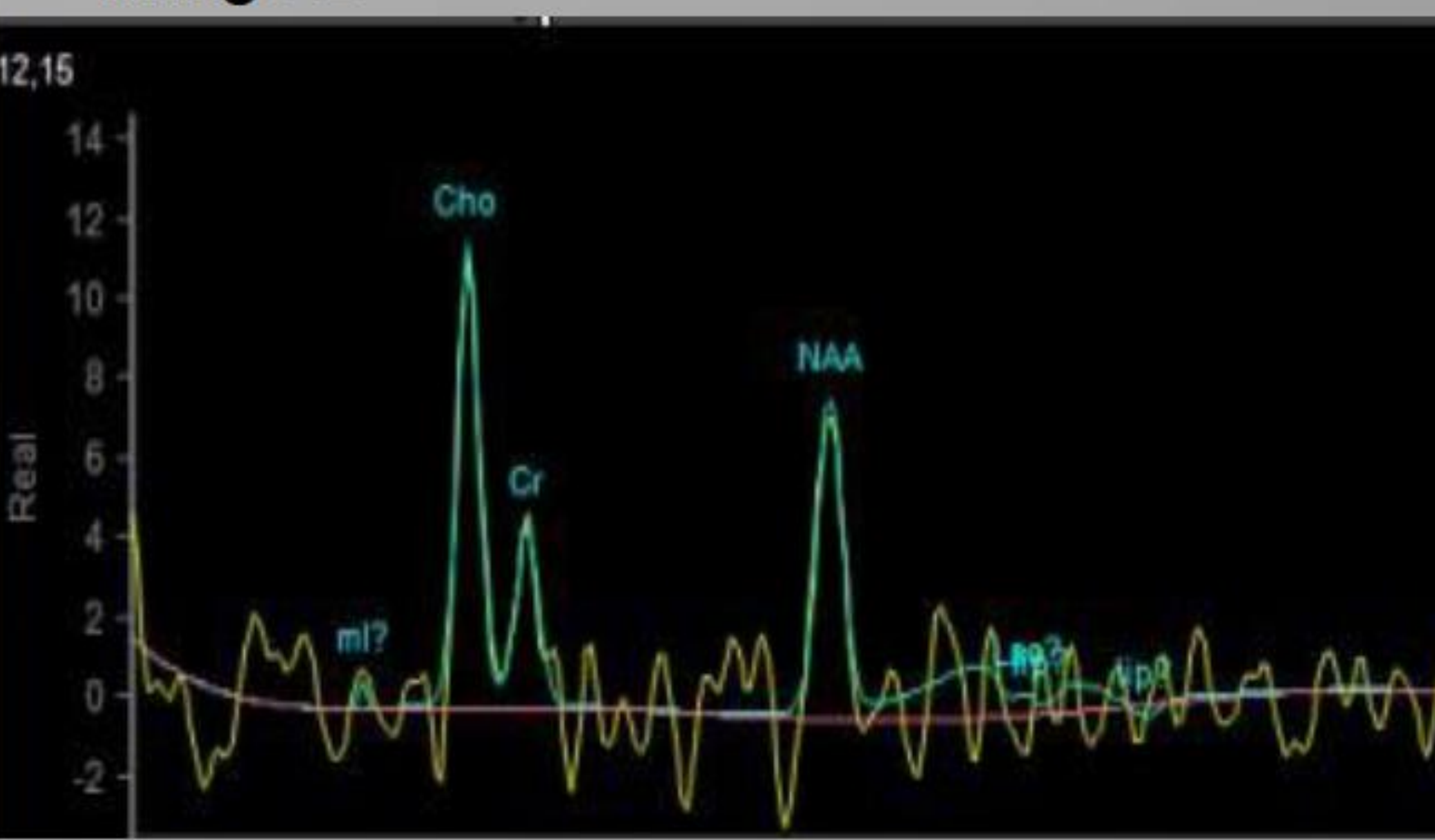

Image 3

Image 4

\section{References}

1Lavabre-Bertrand T, Bourquard P, Chiesa J, Berthéas MF, Lefort G, Taïb J, Lavabre-Bertrand C, Navarro M, Bureau JP. Diabetes insipidus revealing acute myelogenous leukaemia with a high platelet count, monosomy 7 and abnormalities of chromosome 3: a new entity?. Eur $\mathrm{J}$ Haematol. 2001 Jan;66(1):66-9.

\section{Conclusions:}

In conclusion, this is the rare case of reporting coexistance of AML, CDI, panhypopituitarism and hypothalamic lesions probable due to leukemic infiltration. 